\title{
RESEARCH OF ROBOTIC SYSTEMS CONTROL METHODS USING MOTION RECOGNITION TOOLS, MACHINE LEARNING AND SKELETALIZATION ALGORITHMS
}

\author{
Paulius Sakalys \\ Vilniaus Kolegija University of Applied Sciences, Lithuania \\ Loreta Savulioniene \\ Vilniaus Kolegija University of Applied Sciences, Lithuania \\ Dainius Savulionis \\ Vilniaus Kolegija University of Applied Sciences, Lithuania
}

\begin{abstract}
The aim of the research is to develop possible control methods of robotic systems based on the usability of motion detection equipment, skeletalization algorithms and robotic systems, integrating them into the existing test bench by performing compatibility tests. The article reviews the possible motion detection systems, establishing the criteria of applicability in the control of robotic systems, describes the experimental research plan, research stand, discusses the research results and presents summarized conclusions and suggestions for the integration of research results into the educational process.
\end{abstract}

Keywords: machine learning, motion recognition, robotic system, skeletalization algorithms.

\section{Introduction}

Robots and robotic systems have not yet replaced people in disadvantaged areas. Humans are still working in potentially physically hazardous work environments, and there are many areas of activity where a sequence of humanrobot interactions could be implemented - joint work that requires training the robot or robotic system to observe, capture, and accurately reproduce human body movements.

The aim of this study is to implement a robotic system control method based on skeletal methods and machine learning algorithms using digital optical human body motion recognition tools to determine the speed of motion recognition. The article reviews the most commonly used skeletal methods, the principles of operation of digital optical motion recognition devices of the human body, the methodology of their interconnection, and presents the results, conclusions and suggestions of the first simulation tests. 


\section{Literature Review}

Structured recognition of human movement is necessary for robotics movement control training. It is appropriate to use the skeletal method to identify the main human kinematic motion points. In order to select the most appropriate skeletal method, it is necessary to define the concept of skeleton and the issues related to the creation of a skeletal model. Skeletal extraction is important in a variety of areas: image processing, process visualization, navigation, medical research, and computer graphics (Davies, 2017). Some authors often refer to the skeleton as the central axis. The central axis is the set of maximum circles drawn at least between two surface points. A maximal circle is one that is not completely scratched into any other circle within the o surface of the object in question (Rokicki, 2010). In three-dimensional space, instead of circles, draw maximum balls (Formula 1):

$$
S_{r}=\left\{y \in R_{3}, d(x, y)<r\right\},
$$

where $\mathrm{r}$ is the radius of the sphere (or circle in the case of two dimensions); $\mathrm{x} \in$ is the central point when $3 \mathrm{O} \mathrm{R} \subset$; $\mathrm{d}(\mathrm{x}, \mathrm{y})$ - distance between $\mathrm{x}$ and $\mathrm{y}$ points in three-dimensional space. Without any additional operations, the central axis becomes the central surface in three-dimensional space (Carmelo, Acutis, Carrabba, Criscenti, \& Vozzi, 2016). It can be argued that the skeleton is a subset of the central surface (Rokicki, 2010; Paulinas \& Rokicki, 2008). By reviewing the relevant sources of information, two main types of skeletons can be distinguished:

- Geometric skeleton - depicts the center of an object. It does not always have to be one point wide (Fig. 1a). Often the geometric skeleton is called the central axis, so the geometric skeleton does not necessarily have to be one pixel wide, but it always corresponds to the center of the object (Rokicki, 2010).

- Skeleton of curves - a set of connected central lines is called a skeleton of curves (Fig. 1b). The number of skeletal branches does not necessarily coincide with the branches of the skeletal object (Jin, Chen, Hoffman, \& Saha, 2017), so not every curved skeleton is at the same time a topological skeleton. The skeleton of the curves is onedimensional everywhere except for the branching points, because the one-dimensional centerlines intersect at those points. 


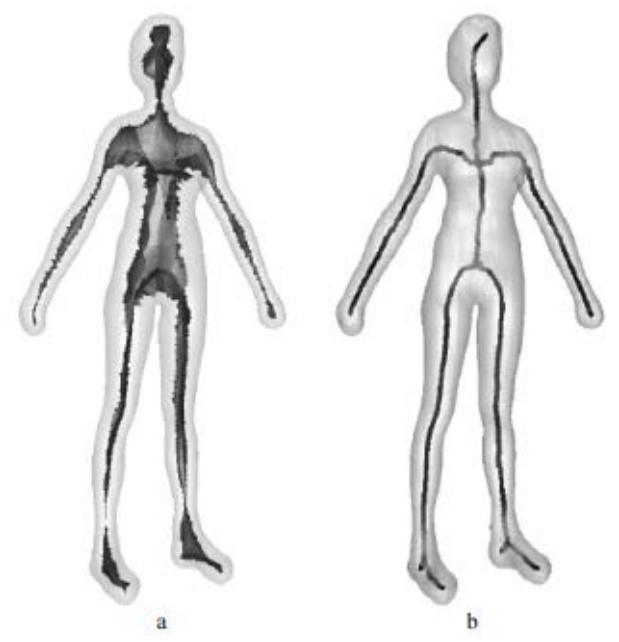

Figure 1 The Main Types of Skeletons (Rokicki, 2010)

Many skeletal extraction (skeletal) algorithms have been developed. Algorithms that implement skeletonization can be divided into the following groups (Saha, Borgefors, \& Baja, 2017):

- Iterative thinning. The main principle of this group of algorithms is the identification and destruction of simple points. Deleting a simple point does not affect the topology of the object. All iterative thinning algorithms operate in discrete space. Thinning iterations are performed until no simple points remain in the object. The main problem with this group of algorithms is limb clipping or uneven reconstruction, so additional conditions are introduced to preserve them. Also, the resulting skeleton is not always geometric, it is centered (Palionyte, 2011). The distance field is formed when each point $P$ of the object $O$ is assigned a distance value to the nearest point $Q$ of the surface $B(O)$ (Formula 2):

$$
\underset{P \in O}{d(P)}=\min _{Q \in B(O)}(d(P, Q))
$$

where $\mathrm{d}(\mathrm{P}, \mathrm{Q})$ is the selected metric. After calculating the distance field, an attempt is made to pave the way through the points where the distance to the boundaries of the object is the maximum. It is these points that correspond to the skeleton of the object (Rokicki, 2010). The aggregation of points is performed using a gradient search or graph theory (searching for the shortest path between the selected maximum). The main advantage of these algorithms is that the distance field is quickly calculated.

- Geometric methods are most commonly used in cases where the surface of an object is represented using a set of scattered points (Chaminade \& 
Cheng, 2019) or a grid of surfaces. These points are used to construct the Voronoi diagram, that is, to create the Voronoi cells. Each wall of a Voronean cell is equidistant from the vertices or points of the grid that constructs it (Fig. 2). The inner edges of Voronoi can be used to approximate the geometric skeleton. The main advantage of these algorithms is speed, because to determine a simple point it is enough to examine the position of its $3 \times 3$ adjacent points (Paulinas \& Rokicki, 2008). Additional transformation steps are required to isolate the onedimensional skeleton.
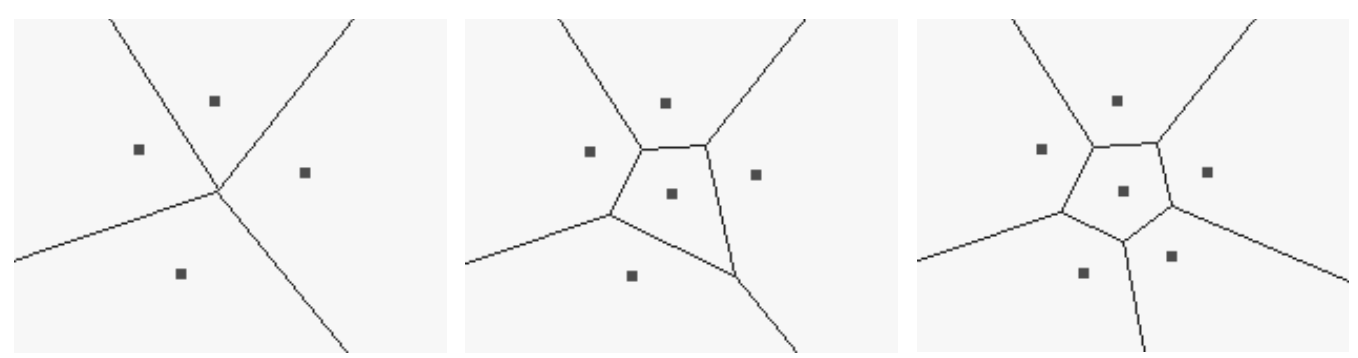

Figure 2 Examples of Voronoy Cells (Palagyi, n.d.)

When creating a skeleton based on points in three-dimensional space, the change of human movement describing the coordinates using digitization means must take into account the calculation speed of the chosen skeletal method (Vassilevski, Olshanskii, Simakov, Kolobov, \& Dailov, 2020). The fastest in terms of calculations is the latter described geometric method.

\section{Methodology}

The description of the research methodology is divided into three parts: description of the research object and its simulation parallelism (part a), explanation of the moving object (human) digital skeleton formation principle (part b) and robotic system motion repetition training algorithm analysis (part c):

a. The object of the study is a robotic system (Fig. 3 a) identified with the human body, having two robotic arms with nine kinematic degrees of freedom manipulators, and a head representing a robot with two degrees of freedom, equipped with environmental video surveillance equipment. For the simulation tests of this study, a virtual kinematic model (Fig. 3 b) was developed, identical to the number of degrees of freedom and motion displacements in space of the constructed robotic system. The initial tests presented in the article will be performed with the right-hand movements of the virtual model. 


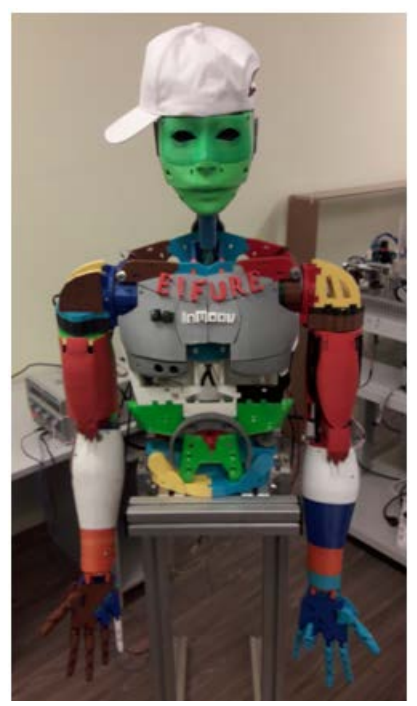

a

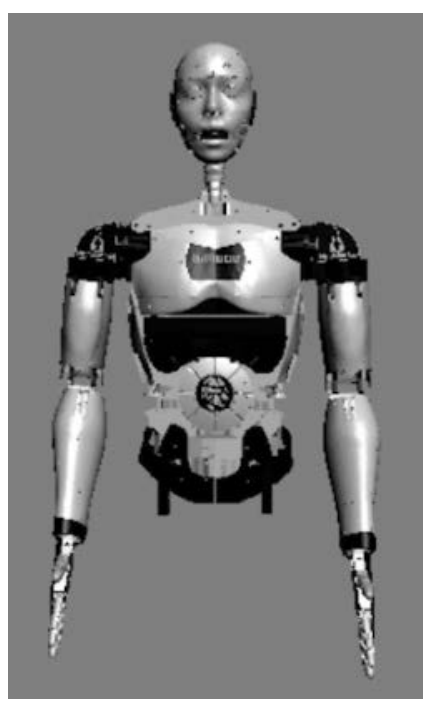

b

Figure 3 Robotic System

b. The principle of digital skeleton formation of a moving object (human): The Kinect multi-infrared projection camera (Fig. 4) is used for the study. (1) and controller (5) (Stitilis, 2014).
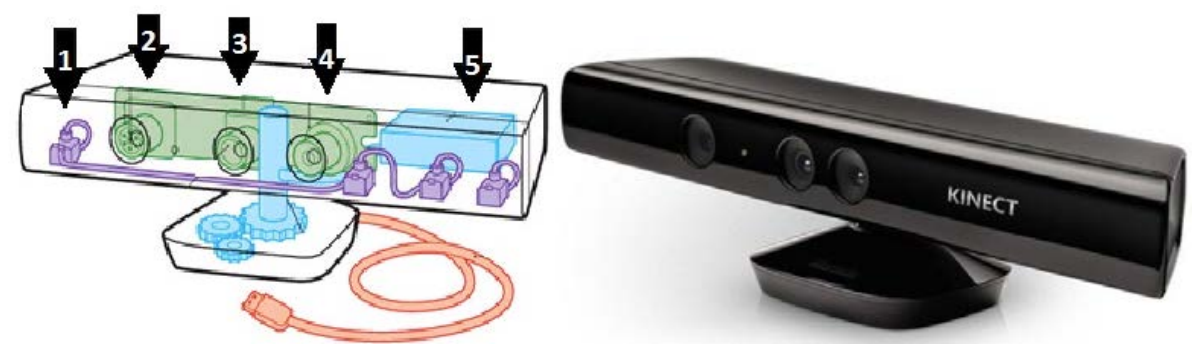

Figure 4 Kinect Multi-Infrared Projection Camera (Stitilis, 2014)

Thanks to the specified cameras and infrared projector, the device forms a three-dimensional image field in which it distinguishes moving points (Fig. 5):

1. The depth camera sensor illuminates the real space and determines the distances specified for each pixel of the image based on the reflections of the infrared point projections.

2. The software that combines the depth camera results and the RGB camera image creates a map of the depth pixel projections of each object in front of the camera, and creates RGB textures based on the location corresponding to the RGB pixels. All information is generated in real time at a rate of 30 times per second.

3. The processed data is sent to the middleware on the receiving computer. 
Sakalys et al., 2021. Research of Robotic Systems Control Methods Using Motion Recognition Tools, Machine Learning and Skeletalization Algorithms

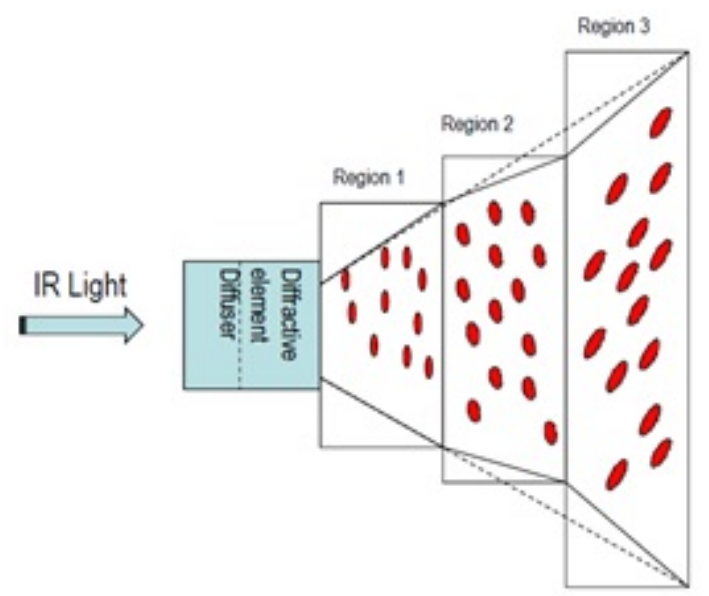

Figure 5 Formation of Three-dimensional Image Field Points by Infrared (Stitilis, 2014)

The first version of the Kinect used for the study returns the joint positions with a three-dimensional vector. The OpenNI library (for access to the cameras) used on the receiving computer provides a function that returns the position of the joint in the orientation matrix (Fig. 6):

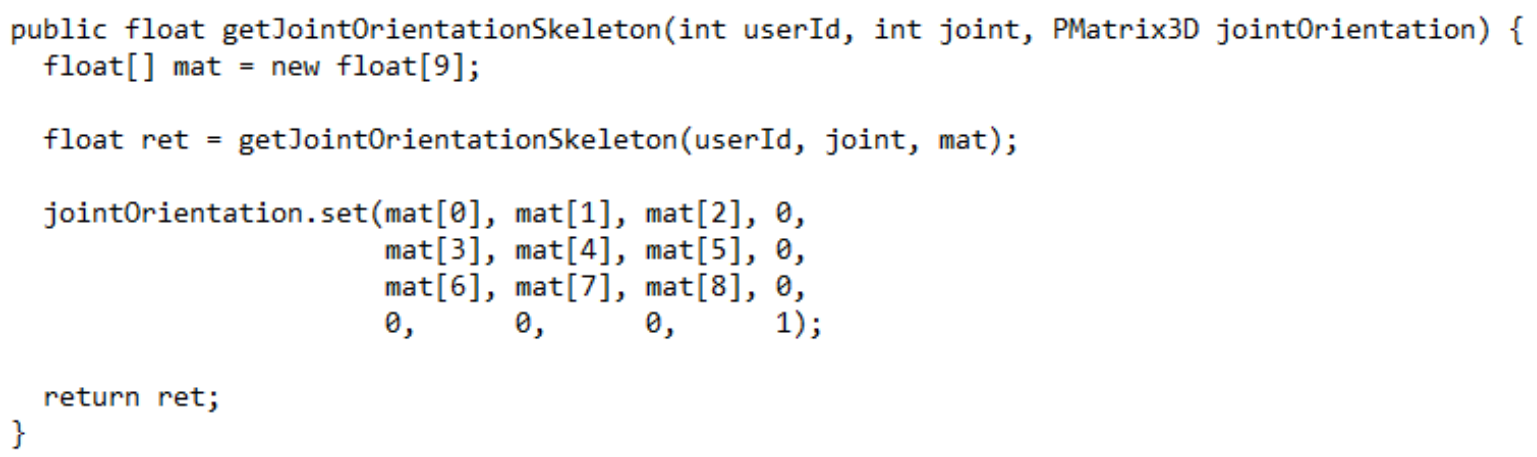

Figure 6 An OpenNI Library Function that Outputs a Position Orientation Matrix

After the calculations, the value of the returned matrix changes only in the first 3 rows. Therefore, we can reduce the size of this matrix to 3x3. Using Euler's characteristics for a given 3x3 matrix (Fig. 7):

$$
R=\left[\begin{array}{lll}
r_{11} & r_{12} & r_{13} \\
r_{21} & r_{22} & r_{23} \\
r_{31} & r_{32} & r_{33}
\end{array}\right]
$$

Figure 7 Transformed Orientation Coordinate Matrix

The steering angles at the three coordinate points are calculated using formulas 1-3:

$$
\begin{gathered}
\theta_{x}=\operatorname{atan} 2\left(r_{32}, r_{33}\right), \\
\theta_{y}=\operatorname{atan} 2\left(-r_{31}, \sqrt{r_{32}^{2}+r_{33}^{2}}\right), \\
\theta_{z}=\operatorname{atan} 2\left(r_{21}, r_{11}\right),
\end{gathered}
$$


SOCIETY. INTEGRATION. EDUCATION

Proceedings of the International Scientific Conference. Volume V, May $28^{\text {th }}-29^{\text {th }}, 2021.448-458$

The coordinates of the obtained moving points are linked to each other to form a skeleton.

c. Robot system motion repetition training algorithm: positions of the obtained skeleton coordinate points - gestures are stored every 30 milliseconds in the control program, giving access rights to call them. Robot system training algorithm (Fig. 8):

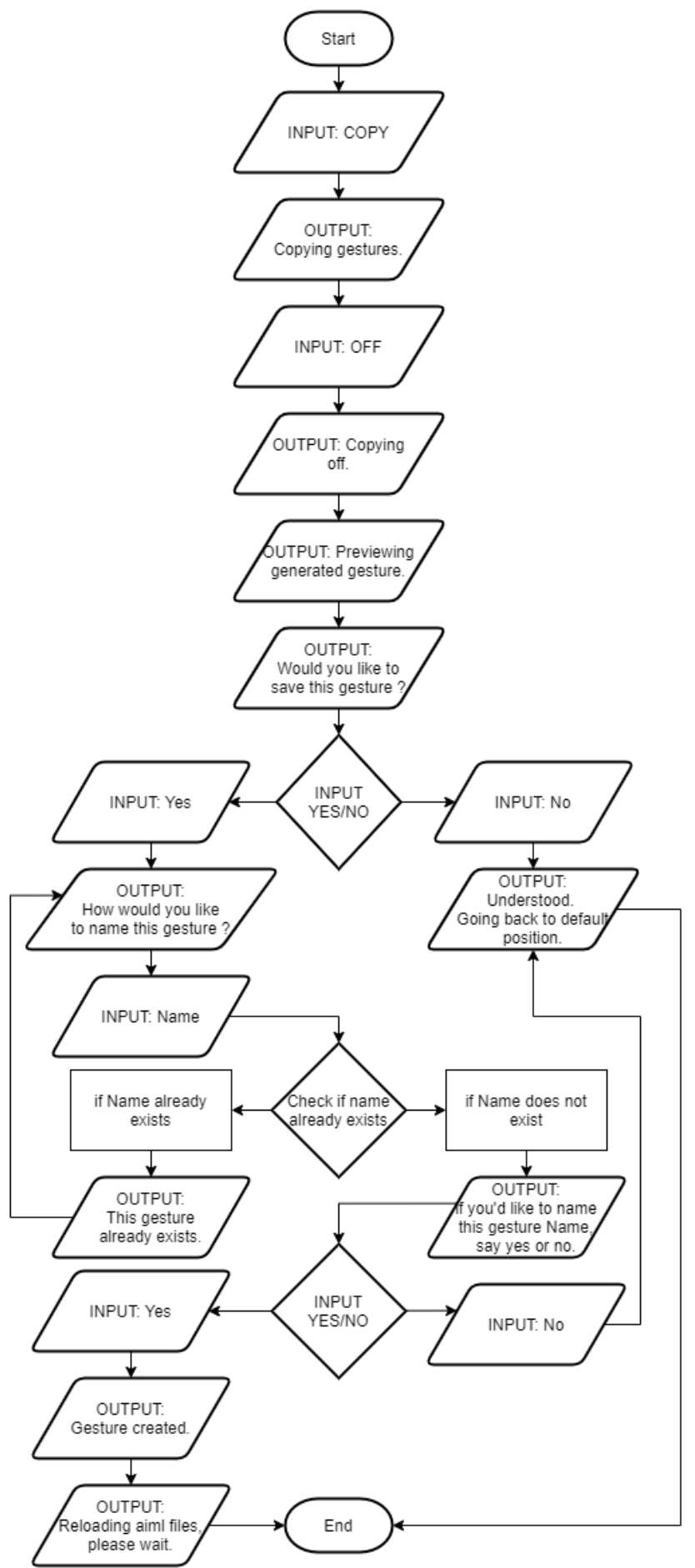

Figure 8 Robot System Training Algorithm 
The sequence of the motion capture function of the Kinect camera is shown in the activity diagram. When using this feature, the client makes a "Copy" request in the command input field or with voice commands. This request starts the repetition of the robot's movements with the Kinect camera. As already mentioned, the positions of the robot servo motors are saved in a new file called "positions" during the entire repetition of the movements. The customer can stop repeating the robot's movements at any time by sending the command "Off". When this is done, the robot repeats the movements again this time taking the positions of the servo drives from the generated file. The user is then asked if they want to save this gesture. For the gesture to be saved successfully, it needs to be given a name. The name must be unique because it is used in two places. The first place is to generate a Python script. This name is used to identify the script file. Also, in order to call the generated method, one of the AIML type files needs to be edited. The AIML interpreter is used to call this function during program operation. AIML is a recursive language based on XML (Nava et al., 2014; Kar, 2011), which allows to parse natural language text input so that it corresponds to the response that a robotic system can send (Fig. 9).

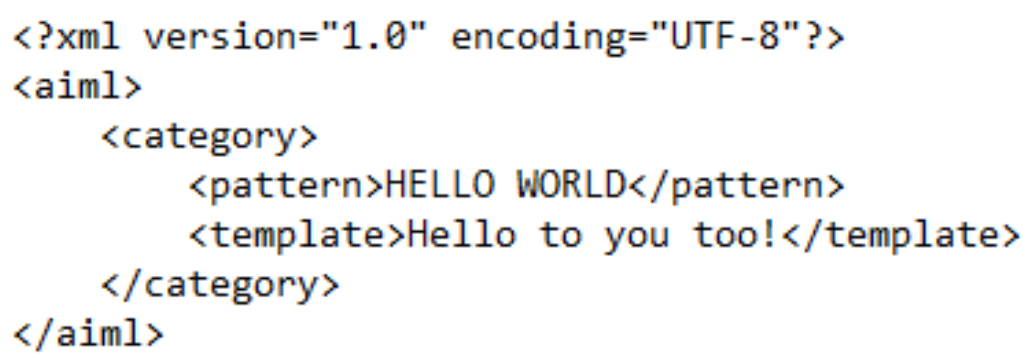

Figure 9 Example of AIML Code Structure

The first "<aiml>" tag indicates the beginning and end of the AIML document. The <category> tag defines the unit of knowledge in the robot database. The number of these tags in AIML-type files is often large, so they simply separate each unique query content, of which we have only one in example 9. Thus the robotic system is trained to identify, record and reproduce the movement of the human body.

\section{Research Results}

Motion recognition and repetition tests were performed with a virtual kinematic model of a robotic system enabled for right-hand control for the first studies. The image of the skeletal projection of the human body and the corresponding movement of the virtual model are mirror images (Fig. 10). Table 
SOCIETY. INTEGRATION. EDUCATION

Proceedings of the International Scientific Conference. Volume V, May $28^{\text {th }}-29^{\text {th }}$, 2021. 448-458

1 shows the average speeds of the steps of skeletal projection construction, which were approximately the same regardless of the position of human movement.

Table 1 Speed Results of Skeletal Projection Creation Stages

\begin{tabular}{|c|c|}
\hline Recognition phase & Time / frame (ms) \\
\hline Face and body recognition & 70 \\
\hline Distance transformation & 8 \\
\hline Point segmentation & 5 \\
\hline Combining points into a skeleton & 10 \\
\hline TOTAL & 93 \\
\hline
\end{tabular}

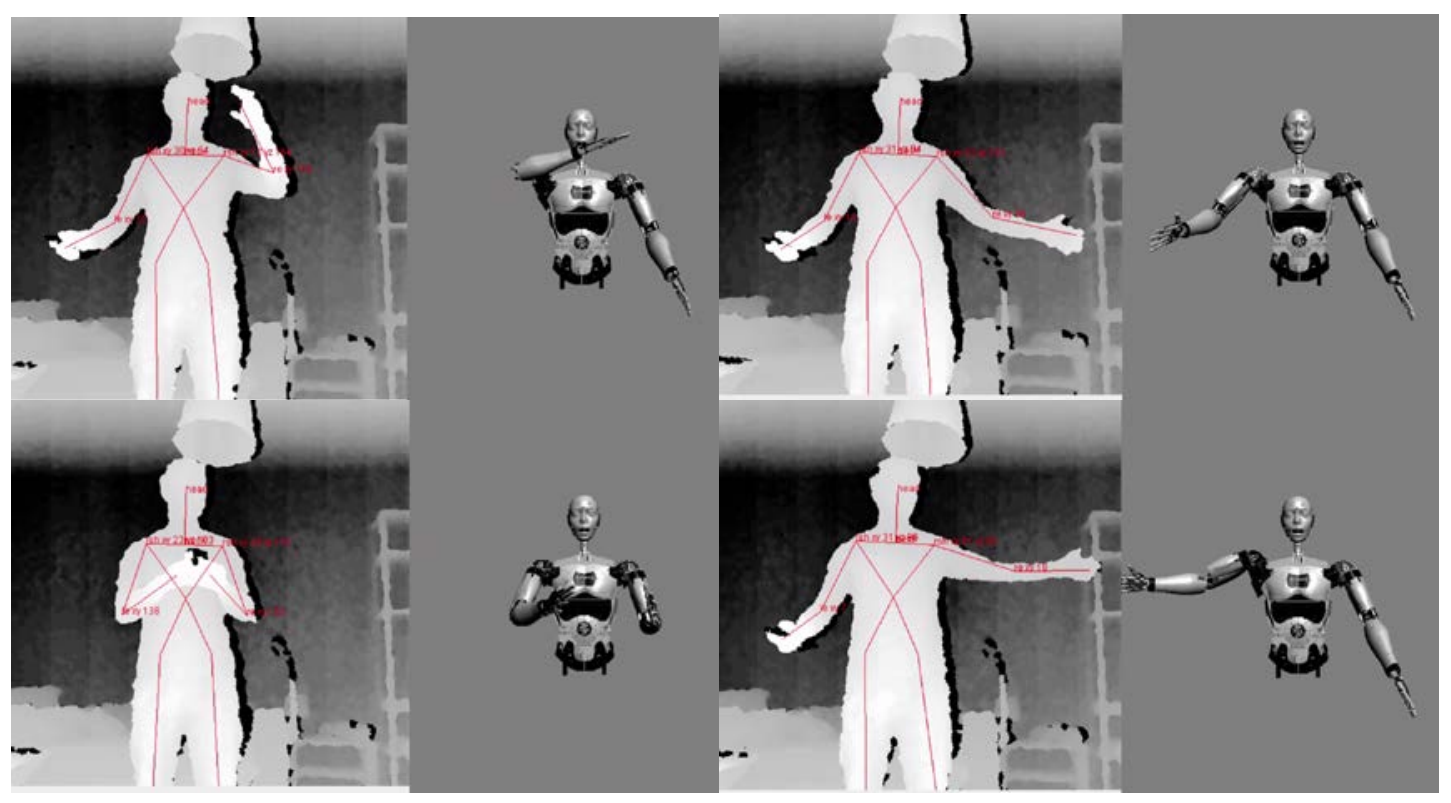

Figure 10 An Image of a Skeletal Projection of the Human Body and a Demonstration of the Repetitive Motion of a Virtual Model (mirrored view)

\section{Conclusions}

The study developed a primary methodology that combines skeletal methods, machine learning algorithms and digital optical equipment to identify human body motion, create a skeletal virtual model describing it in spatial point coordinates, create a coordinate system and save it in a robotic system database with the ability to call when performing motion simulation in a virtual model:

- After reviewing the most suitable skeletalization methods available, the fastest geometric skeletalization method in terms of calculation was selected;

- $\quad$ Kinect digital optical infrared projector used to isolate the human body from the environment; 
Sakalys et al., 2021. Research of Robotic Systems Control Methods Using Motion Recognition Tools, Machine Learning and Skeletalization Algorithms

- The OpenNI library was used for three-dimensional vector processing, and an algorithm for calculating, configuring, recording, and calling motion angles was developed using the AIML software interpreter;

- In the virtual model, the movements of the skeletal projection of the human body are repeated, the average total speed of the formation of the skeletal projection was determined by experiments: 93 ms per frame;

The description of the performed research methodology and the obtained research results can be used in areas where synergistic interaction of human and robotic system synchronous movements is possible: performing dynamic, timevarying functions that do not operate according to a predetermined sequence of actions. The results of the study also have a wide range of applications in the training process: in the study of the properties of machine training, in the development of robotics, control systems for robotic systems and algorithms. Continuing this study, it would be appropriate to combine coordinate motion angle assemblies with a real robotic system and perform motion repeatability and positioning accuracy tests.

\section{References}

Carmelo, M., Acutis, A., Carrabba, M., Criscenti, G., Vozzi, G. (2016). Machine design for multimaterial processing. Nanobiomaterials in Soft Tissue Engineering: Applications of Nanobiomaterials, Volume 5, 111-140. DOI: https://doi.org/10.1016/B978-0-323-42865$1.00005-2$

Chaminade, T., \& Cheng, G. (2019). Social cognitive neuroscience and humanoid robotics. Journal of Physiology - Paris, Volume 103, 286-295. DOI: https://doi.org/ 10.1016/j.jphysparis.2009.08.011

Davies, E.R. (2017). Computer Vision. London: AcademicPress.

Jin, D., Chen, C., Hoffman, E.A., Saha, P.K. (2017). Curve skeletonization using minimumcost path. Skeletonization: Theory, Methods and Applications, 151-180. DOI: https://doi.org/10.1016/B978-0-08-101291-8.00007-9

Kar, A. (2011). Skeletal Tracking using Microsoft Kinect. Kanpur.

Nava, A., Brena, R., Garrido, L. (2014). Recognizing Activities Using a Kinect Skeleton Tracking and Hidden Markov Models. 13th Mexican International Conference on Artificial Intelligence (MICAI). DOI: 10.1109/MICAI.2014.18

Palagyi, K. (n.d.). Skeletonization. Retrieved from: http://www.inf.u-szeged.hu/ palagyi/skel/skel.html

Palionyte, A (2011). Discretization of Continuum Structures via Image Algebra Methods. Vilnius: Vilnius Gediminas Technical University.

Paulinas, M., \& Rokicki, J. (2008). Panasios formos pavirsiu, centrines asies radimo ir filtravimo metodika. Biomedical Engineering, 209-212.

Rokicki, J. (2010). Skeletavimo metodu apzvalga. Science - Future of Lithuania, Vol. 2 (No. 1), 19-22. 
SOCIETY. INTEGRATION. EDUCATION

Proceedings of the International Scientific Conference. Volume V, May $28^{\text {th }}-29^{\text {th }}, 2021.448-458$

Saha, P.K., Borgefors, G., Baja, G.S. (2017). Skeletonization and its applications - a review. Skeletonization: Theory, Methods and Applications, 3-42. DOI: https://doi.org/ 10.1016/B978-0-08-101291-8.00002-X

Stitilis, R. (2014). Kinect Controlled Computer Game. Siauliai: Siauliai University.

Vassilevski, Y., Olshanskii, M., Simakov, S., Kolobov A., Dailov, A. (2020). Computational hemodynamics in vascular surgery. Personalized Computational Hemodynamics: Models, Methods, and Applications for Vascular Surgery and Antitumor Therapy, 189215. DOI: https://doi.org/10.1016/B978-0-12-815653-7.00009-9 